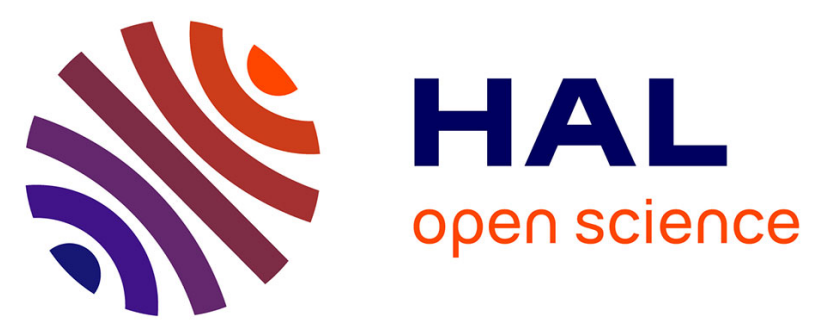

\title{
Effects of 2-D random velocity perturbations on 2-D SH short-period ground motion simulations in the basin of Nice, France
}

\author{
F Tchawe, C Gelis, L Bonilla, Fernando Lopez-caballero
}

\section{- To cite this version:}

F Tchawe, C Gelis, L Bonilla, Fernando Lopez-caballero. Effects of 2-D random velocity perturbations on 2-D SH short-period ground motion simulations in the basin of Nice, France. Geophysical Journal International, 2021, 226 (2), pp.847-861. 10.1093/gji/ggab141 . hal-03200204

\section{HAL Id: hal-03200204 \\ https://hal.science/hal-03200204}

Submitted on 3 Nov 2021

HAL is a multi-disciplinary open access archive for the deposit and dissemination of scientific research documents, whether they are published or not. The documents may come from teaching and research institutions in France or abroad, or from public or private research centers.
L'archive ouverte pluridisciplinaire HAL, est destinée au dépôt et à la diffusion de documents scientifiques de niveau recherche, publiés ou non, émanant des établissements d'enseignement et de recherche français ou étrangers, des laboratoires publics ou privés. 


\title{
Effects of 2-D random velocity perturbations on 2-D $S H$ short-period ground motion simulations in the basin of Nice, France
}

\author{
F.N. Tchawe, ${ }^{1,2}$ C. Gelis, ${ }^{1}$ L.F. BONILLA ${ }^{\circ 2}$ and F. Lopez-Caballero ${ }^{3}$ \\ ${ }^{1}$ Institut de Radioprotection et de Sureté Nucléaire, BP 17, 92262 Fontenay-aux-Roses Cedex, France. E-mail: tflomin@yahoo.com \\ ${ }^{2}$ GERS Department, Université Gustave Eiffel, 77447 Marne-la-Vallée Cedex 2, France \\ ${ }^{3}$ MSSMat Laboratory, CNRS UMR 8579, CentraleSupélec Paris-Saclay Univ., 3 Rue Joliot-Curie, 91190 Gif-Sur-Yvette, France
}

Accepted 2021 March 31. Received 2021 March 30; in original form 2020 July 17

\begin{abstract}
S U M M A R Y
Some geological configurations, like sedimentary basins, are prone to site effects. Basins are often composed of different geological layers whose properties are generally considered as spatially homogeneous or smoothly varying. In this study, we address the influence of small-scale velocity fluctuations on seismic response. For this purpose, we use the spectral element method to model the 2-D $S H$ wave propagation on a basin of $1.1 \mathrm{~km}$ long and $\approx$ $60 \mathrm{~m}$ deep, representing a 2-D profile in the city of Nice, France. The velocity fluctuations are modelled statistically as a random process characterized by a Von Karman autocorrelation function and are superimposed to the deterministic model. We assess the influence of the amplitude and correlation length of the random velocities on the surface ground motion. We vary the autocorrelation function's parameters and compute seismic wavefields in 10 random realizations of the stochastic models. The analyses of our results focus on the envelope and phase differences between the waveforms computed in the random and deterministic models; on the variability of ground motion intensity measures, such as the peak ground velocity, the pseudo-spectral acceleration response; and the 2-D basin response (transfer function). We find that the amplitude of fluctuations has a greater effect on the ground motion variability than the correlation length. Depending on the random medium realization, the ground motion in one stochastic model can be locally amplified or deamplified with respect to the reference model due to the presence of high or low velocity contrasts, respectively. When computing the mean amplification of different random realizations, the results may be smaller than those of the reference media due to the smoothing effect of the average. This study highlights the importance of knowing the site properties at different scales, particularly at small scales, for proper seismic hazard assessment.
\end{abstract}

Key words: Numerical modelling; Statistical methods; Earthquake ground motions; Site effects; Wave propagation.

\section{INTRODUCTION}

It is well known that local site characteristics may produce large ground motion amplifications during earthquakes (e.g. Trifunac \& Lee 1971; Turker et al. 1984; Bard \& Bouchon 1985; Ktenidou et al. 2016). The effect of the local geology on the incoming wavefield is commonly denoted as site effects and can lead to important damages of infrastructures and loss of lives during earthquakes [e.g. the Great Hanshin earthquake as in Pitarka et al. (1998), and the Michoacan earthquake in Anderson et al. (1986)]. Their proper assessment is thus important for accurate seismic hazard evaluations.

Investigating site effects can be done by analysing seismic records or simulating seismic wave propagation in realistic media. Observed seismic data provide a basis for theoretical and experimental research and are of great value to understand site effects. Experimental approaches, like the standard spectral ratio technique, which relies on site-specific transfer functions (Borchedt 1970), are used to evaluate the local site response from earthquake records. However, the scantiness of earthquakes in regions of low to moderate seismicity makes their evaluation difficult and seismologists also use ambient noise vibrations to estimate site effects (e.g. Nakamura 1989; Kanai 1993; Bard 1999; Perron et al. 2018; Tchawe et al. 2019). On the other hand, numerical simulations of seismic wave propagation allow a direct evaluation of the site response, provided that the input motions, velocity, density, and attenuation of the media are well characterized (e.g. Day et al. 2005; Chaljub 
et al. 2010; Moczo et al. 2014). The distribution and characteristics of mechanical properties can be inferred using deterministic geophysical imaging methods (e.g. multichannel analysis of surface waves, seismic reflection, horizontal to vertical spectral ratio, among other techniques), but their limited resolution makes it difficult to map the short-wavelength (or small-scale) variations of the soil properties in the Earth's subsurface. As a result, velocity models often used in ground motion simulations generally assume smooth lateral variations of elastic properties, which may be adequate for long-period ground motion estimations. However, for short-period ground motion simulations, the wavefield can be very sensitive to the small-scale variations in the soil properties (e.g. Hartzell et al. 2010; Emoto et al. 2017; E1 Haber et al. 2019). Furthermore, the need for computing high-frequency seismograms in engineering applications requires the description of short-wavelength variations of the media. Since it is impossible to characterize them totally, they are usually approximated using stochastic approaches (e.g. Frankel 1989; Hong \& Kennett 2003; Sato \& Fehler 2012).

Understanding the effect of variations in velocity models (henceforth regarded to as random velocity heterogeneities) on the simulated ground motion is a topic under active investigation in seismology. Most studies that consider random velocity heterogeneities often focus on comprehending seismic scattering in the Earth's crust (e.g Frankel 1989; Holliger \& Levander 1992; Roth \& Korn 1993; Frenje \& Juhlin 2000; Hong \& Kennett 2003; Imperatori \& Mai 2013). Other studies have investigated the random velocity heterogeneities effects on surface ground motion. For example, Hartzell et al. (2010) observed that including them in regional ground motion simulations in the San Francisco Bay Area lead to significant deviations in the predicted ground velocities for standard deviations of 5 to 10 per cent of the random media. Imperatori \& Mai (2015) and Iwaki et al. (2018) also observed that random velocity heterogeneities lead to large variability in the simulated ground motion parameters.

These studies deal with crustal velocity heterogeneities whose characteristic scales are larger than those observed in sediments. Modelling velocity heterogeneities at the sedimentary scale can be challenging, and few studies have been targeted towards understanding their effect on surface ground motion. Thompson et al. (2009) observed that for wave propagation at shallow depths $(\leq 200 \mathrm{~m})$, random velocity heterogeneities must exhibit large fluctuations (approximately 25 per cent of the mean or greater) to reproduce the observed spectral amplification of the ground motion. Pagliaroli et al. (2014) showed that velocity heterogeneities could locally double the Housner intensity of ground motion computed at Rome's Central Archaeological Area. Stripajova et al. (2018) showed that considering random heterogeneities in 2-D ground motion simulations increases the cumulative absolute velocity's aggravation factor. El Haber et al. (2019) observed that the ground motion indicators are mostly sensitive to the coefficient of variation of the velocity heterogeneities, producing large spatial variability.

In this study, we aim to understand the effect of random velocity heterogeneities on the computation of surface ground motion from a site-effect analysis point of view. We use a 2-D velocity model of the Nice (France) sedimentary basin, which is perturbed, to perform 2-D $\mathrm{SH}$ viscoelastic numerical simulations of seismic wave propagation. We introduce random velocities in the sedimentary basin as 2D spatially correlated random processes characterized by a Von Karman autocorrelation function (ACF). We investigate the role of the correlation length and the strength of the velocity fluctuations on the simulated ground motion. The area of study is introduced in Section 2 . The stochastic generation of random velocity models and
Table 1. Lithology and mechanical characteristics of the nine layers of the 3-D geotechnical model of Nice. The layers are denoted by codes $1-9$. Code $\mathrm{R}$ represents the seismic bedrock

\begin{tabular}{llccc}
\hline Code & Soil lithology & $\begin{array}{c}\rho(\mathrm{Kg} \\
\left.\mathrm{m}^{-3}\right)\end{array}$ & $\begin{array}{c}V_{\mathrm{s}}(\mathrm{m} \\
\left.\mathrm{s}^{-1}\right)\end{array}$ & $\begin{array}{c}V_{\mathrm{p}}(\mathrm{m} \\
\left.\mathrm{s}^{-1}\right)\end{array}$ \\
\hline 1 & Embankments, topsoil, concrete & 1900 & 180 & 440 \\
2 & Sand, pebbles, gravel & 1900 & 290 & 710 \\
3 & Silt, clay & 1700 & 200 & 489 \\
4 & Sand, gravel, pebbles & 2100 & 330 & 808 \\
5 & Fine sand, silty sand & 1800 & 250 & 612 \\
6 & Sand, gravel, pebbles & 2100 & 300 & 734 \\
7 & Silt, clay & 1800 & 220 & 538 \\
8 & Sand, gravel, pebbles & 2000 & 290 & 710 \\
9 & Screes & 2000 & 300 & 734 \\
$\mathrm{R}$ & Bedrock & 2100 & 1000 & 2449 \\
\hline
\end{tabular}

numerical solution of the wave propagation are presented in Section 3. Analysis of the sensitivity of the ground motion to the variability of the random media is described in Section 4. We discuss the influence of the velocity fluctuations on the ground motion for site effects assessment in Section 5. We finish by providing conclusions and perspectives to the current study in Section 6 .

\section{THE NICE (FRANCE) SEDIMENTARY BASIN AND 2-D VELOCITY MODEL}

The city of Nice is a densely populated area and one of the most seismically active regions in France (e.g Courboulex et al. 2007). The city is built on three major geological units: (1) Quaternary alluvial deposits originating mainly from the Var and Paillon rivers, (2) conglomerates of the Pliocene age which form the western hills of the region and (3) Jurassic and Cretaceous bedrock. Geomorphologically, the area comprises reliefs, valleys and sedimentary fillings, which are prone to site effects. The region has been the subject of different studies, like the GEMITIS (Arnal \& Martin 1998) and GEMGEP (Bard et al. 2005) projects, which provided a detailed geological and geotechnical description of the subsurface for site effect analysis and seismic hazard assessment purposes. These led to developing a 3-D geotechnical model of the region using borehole data and ambient noise measurements (Bertrand et al. 2007). The model has nine geological layers overlying a bedrock. The lithology and mechanical characteristics of the different layers are summarized in Table 1. Fig. 1 shows the digital elevation model of city. The black line along the central valley shows the 2-D vertical cross-section of the region's 3-D geotechnical model, which we use for the 2-D numerical simulations in this work. The cross-section passes through seven of nine layers in the 3-D model, and layers 4 and 9 in Table 1 are absent. The geometry and 2-D velocity mode1 of the sedimentary basin comprising the computational domain is shown in Fig. 2 and is denoted as model M.

\section{METHODS}

\subsection{Stochastic modelling of random heterogeneities}

The mathematical description of random media for the modelling of random heterogeneities has widely been described in several publications (e.g Goff \& Jordan 1988; Klimes 2002; Sato \& Fehler 2012). In this section, we briefly summarize the essential theory and steps for the generation of random fields. The heterogeneous 


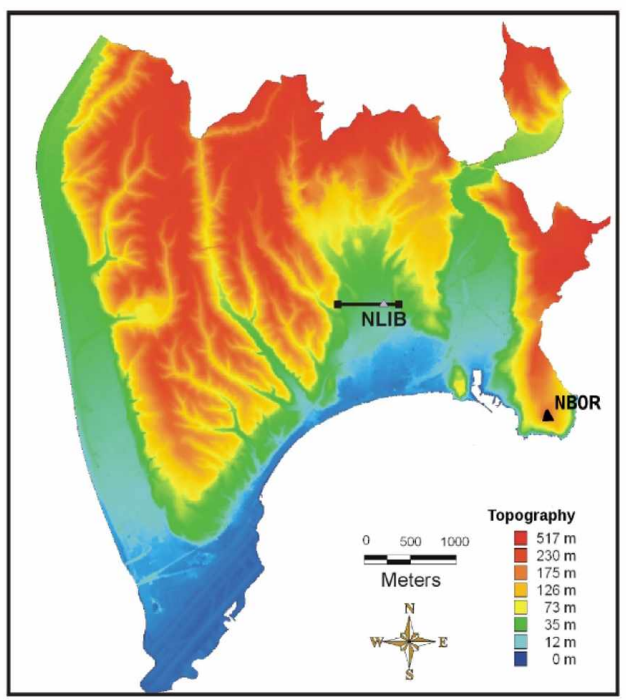

Figure 1. Digital elevation model of the Nice (France) region displaying the reliefs and valleys. The black line shows the position along the Paillon valley where the vertical 2-D cross-section of the velocity model used in this study was obtained (after Peyrusse et al. 2013).

velocity (v) of the soil can be expressed as a function of space as

$v(\boldsymbol{x})=v_{0}(1+\epsilon(\boldsymbol{x}))$

where $v_{0}$ is the mean compressional $V_{\mathrm{P}}$ or shear wave velocity $V_{\mathrm{S}}$ of the medium and $\epsilon(\boldsymbol{x})=\delta v(\boldsymbol{x}) / v_{0}$ is the fractional fluctuation of the wave velocity. $\epsilon(\boldsymbol{x})$ is a random function of space with mean value being zero $(\langle\epsilon(\boldsymbol{x})\rangle=0)$. We express the spatial coordinates in 2-D Euclidean space $\boldsymbol{x}=(x, z)$, where $x$ and $z$ denote the horizontal and vertical directions of space, respectively.

The ACF, $C(\boldsymbol{x})$, of the fractional velocity fluctuation can be used to characterize the stochastic nature of the random media. When the randomness is stationary and isotropic, the ACF is a function of the spatial lag $(r \equiv|x|)$ distance only. Several types of ACFs of random media have been developed for geophysical applications and wave propagation problems (e.g. Ishimaru 1978; Klimes 2002).

Few examples in the literature provide direct empirical observations of the spatial correlation structure of seismic properties. As a result, the correlation function is often constrained by observations of seismic phenomena. For example, Nakata \& Beroza (2015) used dense-array recordings of the ambient seismic wavefield to constrain the random-field model representations of a 3-D $P$-wave velocity model in Long Beach, California. In their study, the Von Karman ACF best fits the imaged $P$-wave velocity model. Several past studies in seismology (e.g. Frankel 1989; Hartzell et al. 2010; Sato \& Fehler 2012; Imperatori \& Mai 2015) also consider the Von Karman ACF when modelling the Earth's heterogeneities. Furthermore, Thompson et al. (2007) measured the spatial horizontal correlation function of the near-surface sediments in the San Francisco Bay area. They observed that the horizontal correlation structure of the average $S$-wave velocity of the upper $10 \mathrm{~m}$ of the soil exhibited an exponential correlation function with a range of about $3 \mathrm{~km}$. The exponential correlation function is a Von Karman ACF with a Hurst number of 0.5 . In this study, we assume that the spatial distribution of velocity heterogeneities also follows a Von Karman correlation function.
The 2-D analytical expression of the Von Karman ACF is expressed in eq. (2) (Goff \& Jordan 1988; Carpentier \& RoyChowdhury 2007) as

$C(r)=\frac{r^{v} K_{v}(r)}{2^{v-1} \Gamma(\nu)}$

where $r$ is the weighted radial autocorrelation lag $\left(\sqrt{x^{2} / a_{x}^{2}+z^{2} / a_{z}^{2}}\right), v$ is the Hurst number, $K_{v}(r)$ is the second modified Bessel function of fractional order $v$ and $\Gamma$ is the Gamma function. The corresponding power spectrum of the Von Karman ACF (eq. 2) is expressed by

$P(k)=\frac{4 \pi v a_{x} a_{z}}{2^{v-1} \Gamma(v)\left(1+k^{2}\right)^{v+1}}$,

where $a_{x}$ and $a_{z}$ are the horizontal and vertical correlation lengths, respectively; $k$ is the weighted radial wavenumber $(k=$ $\sqrt{k_{x}^{2} a_{x}^{2}+k_{z}^{2} a_{z}^{2}}$, with $k_{x}, k_{z}$ being the horizontal and vertical wavenumbers, respectively).

The random media are generated by applying a 2-D uniform random phase to the 2-D power spectrum (eq. 3) and then transforming back to the space domain using the inverse 2-D Fourier transform. The resulting random field is normalized to have zero mean and unit variance. The random field is then scaled to the desired root-meansquare (RMS) fluctuation using a given coefficient of variation $\left(c_{v}\right)$.

In this work, we generated random fields of the shear wave velocities. All the other material parameters are considered constant. We truncated the random velocity distributions between $-2 \sigma$ and $2 \sigma$ (with $\sigma$ being the standard deviation) to limit the long tail normal distribution. Truncating the distribution of velocities also avoids having negative velocities. It eases the meshing process by avoiding very fine grids due to low-velocity values, thereby ensuring the stability and reducing the computational demands of the numerical scheme. Other studies may use different distributions for the velocity field (e.g. Thompson et al. (2009) used the beta distribution to characterize the fluctuation of velocity). Moreover, El Haber et al. (2019) observed that simulated ground motion is less sensitive to the velocity probability distributions compared to other parameters of the random medium (correlation length and coefficient of variation). Assessing which distribution best describes the media is not the purpose of this study.

\subsection{Generation of the 2-D random velocity models}

We consider random velocity perturbations only within the sedimentary basin to investigate their effect on the computed ground motion. The bedrock is assumed to have a uniform $V_{\mathrm{S}}$ of $1000 \mathrm{~m} \mathrm{~s}^{-1}$. Since we conduct a parametric analysis, the statistical parameterscorrelation length $(a)$, Hurst exponent $(v)$ and the coefficient of variation $\left(c_{v}\right)$-that control the correlation function are chosen based on values reported in the literature. Some studies at the crustal-scale (e.g. Frankel 1989) report correlation lengths of the order of $10 \mathrm{~km}$ and coefficient of variation in the range of 5-10 per cent in order to replicate teleseismic traveltime anomalies and seismic coda at frequencies up to $30 \mathrm{~Hz}$. Correlation lengths of these orders are unrealistic for small-dimension sedimentary basins. Considering correlation lengths, which are orders of magnitude larger than the medium's size, will have imperceptible effects on the wave propagation in shallow depths, especially for short wavelengths. At the sedimentary scale, Thompson et al. (2009) observed that the model must exhibit large coefficients of variations, around 25 per cent or larger, and correlation lengths of $\approx 100 \mathrm{~m}$ to reproduce the empirical transfer functions at the OKYH07 site of the Kiban-Kyoshin 


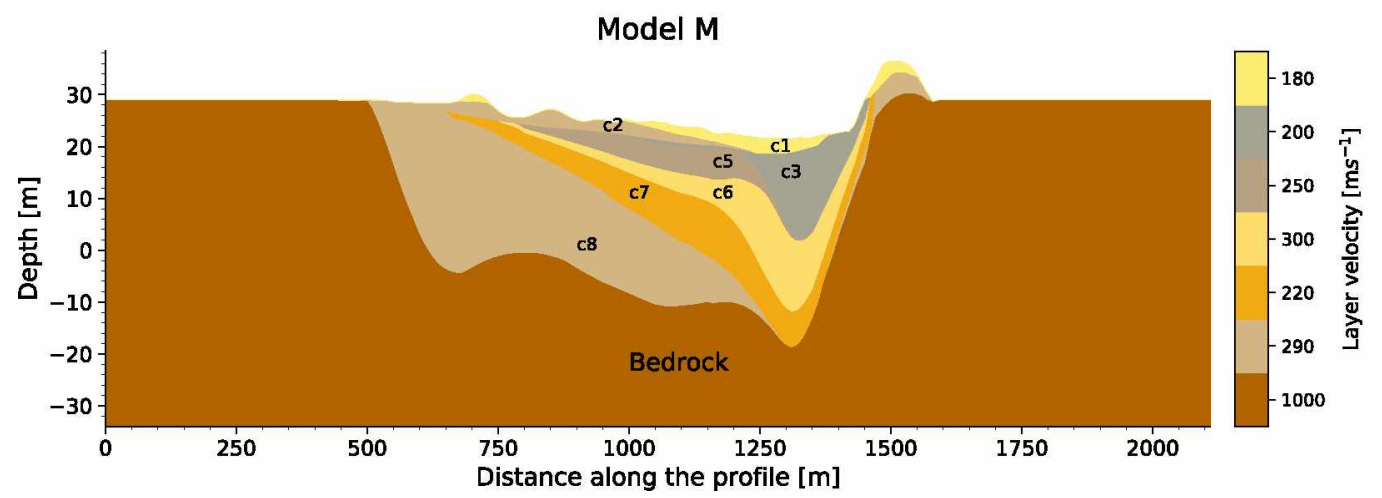

Figure 2. 2-D velocity model (M) of the Nice sedimentary basin, labels c1-c8 correspond to the layer codes listed in Table 1.

network in Japan. table 4 of Thompson et al. (2009) summarizes the $S$-wave velocity $c_{v}$, reported by Holzer et al. (2005) and Wills \& Clahan (2006), of sedimentary units. The $c_{v}$ values vary in between 14 and 46 per cent. Furthermore, table 2 of Sato (2019) summarizes the different statistical parameters of the Von Karman spectrum proposed and measured in the literature. These summaries provide useful insights into the order of the statistical parameters exhibited by different geologic materials.

In this study, we simulate seismic wavefields in a shallow basin $(<60 \mathrm{~m})$. For scattering to be significant in this range, the order of the vertical correlation length must be less than or equal to the depth of the sedimentary basin. The minimum and maximum $V_{\mathrm{S}}$ in the deterministic velocity model are 180 and $300 \mathrm{~m} \mathrm{~s}^{-1}$, respectively. The source time function's dominant frequency is $6 \mathrm{~Hz}$ (Fig. 3), which gives dominant wavelengths between 30 and $50 \mathrm{~m}$. We chose two values of the correlation length, 10 and $50 \mathrm{~m}$. The correlation length of $10 \mathrm{~m}$ is smaller than the dominant wavelengths, while the correlation length of $50 \mathrm{~m}$ falls in the range of the dominant wavelength. We also choose three values for the $c_{v}, 5,10$ and 30 percent to reflect low, intermediate and high velocity fluctuations in the sedimentary basin. These three values of $c_{v}$ fall in the interval of values commonly reported in the literature for sediments (e.g. Thompson et al. 2009; Sato 2019).

Using a combination of these parameters, we build five 2-D random media (R1-R5) whose characteristics are summarized in Table 2. All five random models are assumed to be isotropic such that vertical and horizontal correlation lengths coincide $\left(a_{x}=a_{z}\right)$. Although sediments generally exhibit larger scale lengths in the horizontal direction than vertical direction due to their formation process, we decided to use the same vertical and horizontal correlation lengths in our models for parametric analysis and modelling simplifications.

The Hurst exponent, $v$, commonly varies between 0 and 0.5 and controls the contribution of short-wavelengths in the medium. Low values increase the variability across high spatial frequencies. We assumed a constant value of $v=0.3$ for all random models. Models R1 and R2 have coefficients of variation of 5 per cent with correlation lengths of 10 and $50 \mathrm{~m}$, respectively, while models R3 and R4 have a coefficient of variation of 30 per cent with correlation lengths of 10 and 50 meters, respectively. Model R5 has a correlation length of $10 \mathrm{~m}$ and a coefficient of variation of 10 percent. This model is an intermediate case between R1/R2, and R3/R4 cases. We also assume that all layers have the same random media characteristics to avoid abrupt changes in velocity at the layer's interface. For each random model, we generate 10 realizations with different random seeds for further statistical analysis.

\subsection{Numerical simulation of seismic wave propagation}

We compute 2-D $S H$ seismic wavefields using the spectral element solver SEM2DPACK (Ampuero 2003). The computational domain is $2100 \mathrm{~m}$ wide and $70 \mathrm{~m}$ deep, as shown in Fig. 2. An unstructured mesh containing 3195 elements with a maximum grid resolution of $10 \mathrm{~Hz}$ is generated, taking into account all model interfaces using an external mesh generator software Trelis 16.4 (Trelis 2019). The maximum element size in the basin is $\approx 1 \mathrm{~m}$. The seismic wavefield is integrated on the mesh domain using seven Gauss-LobattoLegendre (GLL) integration points. The minimum GLL separation distance in the basin's spectral element grid is $\approx 0.1 \mathrm{~m}$. We mimic an infinite lateral domain using periodic boundary conditions. Periodic boundaries were adequately used by Peyrusse et al. (2013) to simulate the basin's response. We apply absorbing boundary conditions at the bottom of the model and impose vanishing traction at the surface. Intrinsic attenuation is taken into account by frequencyindependent $Q$ values between 5 and 5000 for a frequency range between 0.01 and $50 \mathrm{~Hz}$ (Liu \& Archuleta 2006), which is based on the rheological model of the Generalized Maxwell body (Emmerich $\&$ Korn 1987). This realistic attenuation was implemented in the spectral element solver by Oral et al. (2019). For the simulations, we define an $S$-wave quality factor $\left(Q_{\mathrm{s}}\right)$ of 50 . The bedrock is assumed to be purely elastic and homogeneous.

We simulate $S H$ wave propagation ( $S$-waves polarized out-ofplane) generated by a vertical incident plane wave. As a source time function, we use a truncated Gaussian wavelet with a central frequency of $6 \mathrm{~Hz}$ (Fig. 3). The seismic wavefield is computed up to $10 \mathrm{~s}$ using a leap-frog time scheme with a calculation time step of $20 \mu$ s. The velocity wavefield at the free surface is recorded every $10 \mathrm{~ms}$ by 420 surface receivers with an interreceiver spacing of $5 \mathrm{~m}$.

We compute seismic waves in both the deterministic and random media. To include the random velocity heterogeneities into the velocity model (Fig. 2), we interpolate the regular grid of the random media over the spectral element mesh of the sedimentary basin using a nearest-neighbounr interpolation algorithm. The random media R1-R5 were all generated on a rectangular grid of $1200 \mathrm{~m} \times$ $250 \mathrm{~m}$ with a constant grid step of $0.2 \mathrm{~m}$ in both space directions.

To ease the writing and reading of this paper, simulations in the reference medium are denoted as $M$, whereas simulations in the random media interpolated over the reference medium are labelled MR1 to MR5 in the text.

Fig. 4 shows examples of one realization of each random model. In MR1, MR2 and MR5 (Figs 4a, b and e, respectively), the initial layering of the sedimentary layers are still preserved after perturbation. On the contrary, models MR3 and MR4 (Figs 4c and d, respectively) with $c_{v}=30$ per cent, the layering structure becomes 

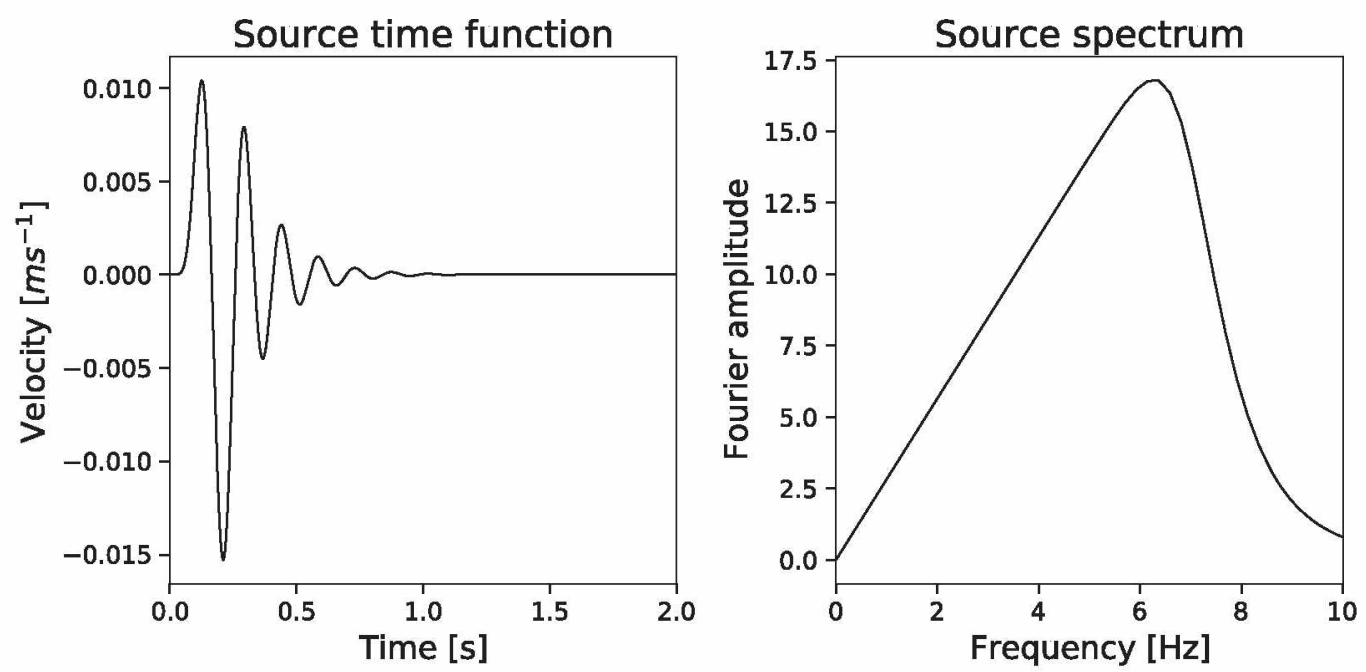

Figure 3. Left: source time function (a truncated Gaussian wavelet). Right: source spectrum showing a dominant frequency of $6 \mathrm{~Hz}$ (after Peyrusse et al. 2013).

Table 2. Characteristics of the random media used to model velocity heterogeneities in the sedimentary basin. $a_{x}$ and $a_{z}$ are the horizontal and vertical correlation lengths respectively, $v$ is the Hurst exponent, and $c_{v}$ is the coefficient of variation.

\begin{tabular}{lccccc}
\hline & \multicolumn{5}{c}{ Random Media } \\
\cline { 2 - 6 } & $\mathrm{R} 1$ & $\mathrm{R} 2$ & $\mathrm{R} 3$ & $\mathrm{R} 4$ & $\mathrm{R} 5$ \\
\hline$a_{x}(\mathrm{~m})$ & 10 & 50 & 10 & 50 & 10 \\
$a_{z}(\mathrm{~m})$ & 10 & 50 & 10 & 50 & 10 \\
$v$ & 0.3 & 0.3 & 0.3 & 0.3 & 0.3 \\
$c_{v}(\%)$ & 5 & 5 & 30 & 30 & 10 \\
\hline
\end{tabular}

almost invisible due to the high intensity of the velocity perturbations.

\section{RESULTS}

To reveal the differences in the wave propagation and ground motion induced by the random media, we compare the results of simulations from the random media to those from the deterministic model M. First, we analyse the differences of waveforms by quantifying the relative discrepancies in phase and amplitude between the deterministic and random media seismic signals. We then quantify their effect on the ground motion in terms of peak ground velocity (PGV), transfer functions (TF), and the pseudo-spectral acceleration (PSA), which are intensity measurements classically used in earthquake engineering.

\subsection{Wave propagation in random heterogeneous media}

Fig. 5(a) displays the velocity wavefield recorded at the surface of the reference model $\mathrm{M}$. The horizontal axis denotes the position of the receivers, which can be directly mapped to the basin structure shown in the bottom panel. This figure displays the complexity of the wave propagation within the sedimentary basin due to the presence of different soil layers, basin geometry, and a strong impedance contrast between the sediments and the underlying bedrock. We show the first $5 \mathrm{~s}$ of propagation, where the stronger part of the ground motion concentrates.
The seismograms recorded at the bedrock contain only the reflections at the free surface because the medium has no layering and a uniform velocity. For the receivers located within the basin (between 500 and $1500 \mathrm{~m}$ ), we can observe strong reflected waves from the edges and propagating across the basin. In particular, the wavefield between 1250 and $1500 \mathrm{~m}$ has higher amplitudes, from 0.4 to $2 \mathrm{~s}$, due to the local trapping of seismic waves in the low-velocity layers $\mathrm{c} 1$ and $\mathrm{c} 3$.

Figs 5(a)-(e) show examples of wavefields from one realization of the random models MR1 to MR5, respectively. Models MR1 and MR2, with a $c_{v}$ of 5 per cent, have similar wavefields and close to the reference case because of the low fluctuation level of random heterogeneities. These models still preserve the initial impedance structure of the basin layers. The wavefield in MR5, with $c_{v}$ of 10 percent, is slightly more scattered than MR1 and MR2. The overall wave propagation in MR5 still remains similar to the reference case as the deterministic model still remains the dominant feature. Conversely, models MR3 and MR4, with $c_{v}=30$ per cent, present notable differences compared to the reference model. The reflected waves at the basin edges are completely scattered by the strong heterogeneities as they propagate across the basin. The high $c_{v}$ value of these models considerably changes the initial velocity profile of the media. For a constant coefficient of variation, a change in the correlation length has little effects on the wave propagation. This is due to the fact that the basin is not large enough for the wave propagation to be affected by the correlation length of the random media. Hence, media MR1 and MR2 have similar seismograms. These results suggest that local heterogeneities do strongly affect the seismic wavefield in their vicinity.

\subsection{Time-frequency analysis: envelope and phase changes}

We quantify the phase and amplitude differences between the computed velocity wavefields in the random media and the reference medium using the single-value envelope (EM) and phase (PM) misfit as the difference measure following Kristekova et al. (2006). First, the seismograms were bandpass filtered between 0.1 and $10 \mathrm{~Hz}$ using a fourth-order non-causal Butterworth filter. The quantities EM and PM vary between 0 and 1 , with values close to 0 implying low differences between the signals, and those close to 1 indicating high 
(a)

$$
a_{x=z}=10 \mathrm{~m}, c_{v}=5 \%(\mathrm{MR} 1)
$$

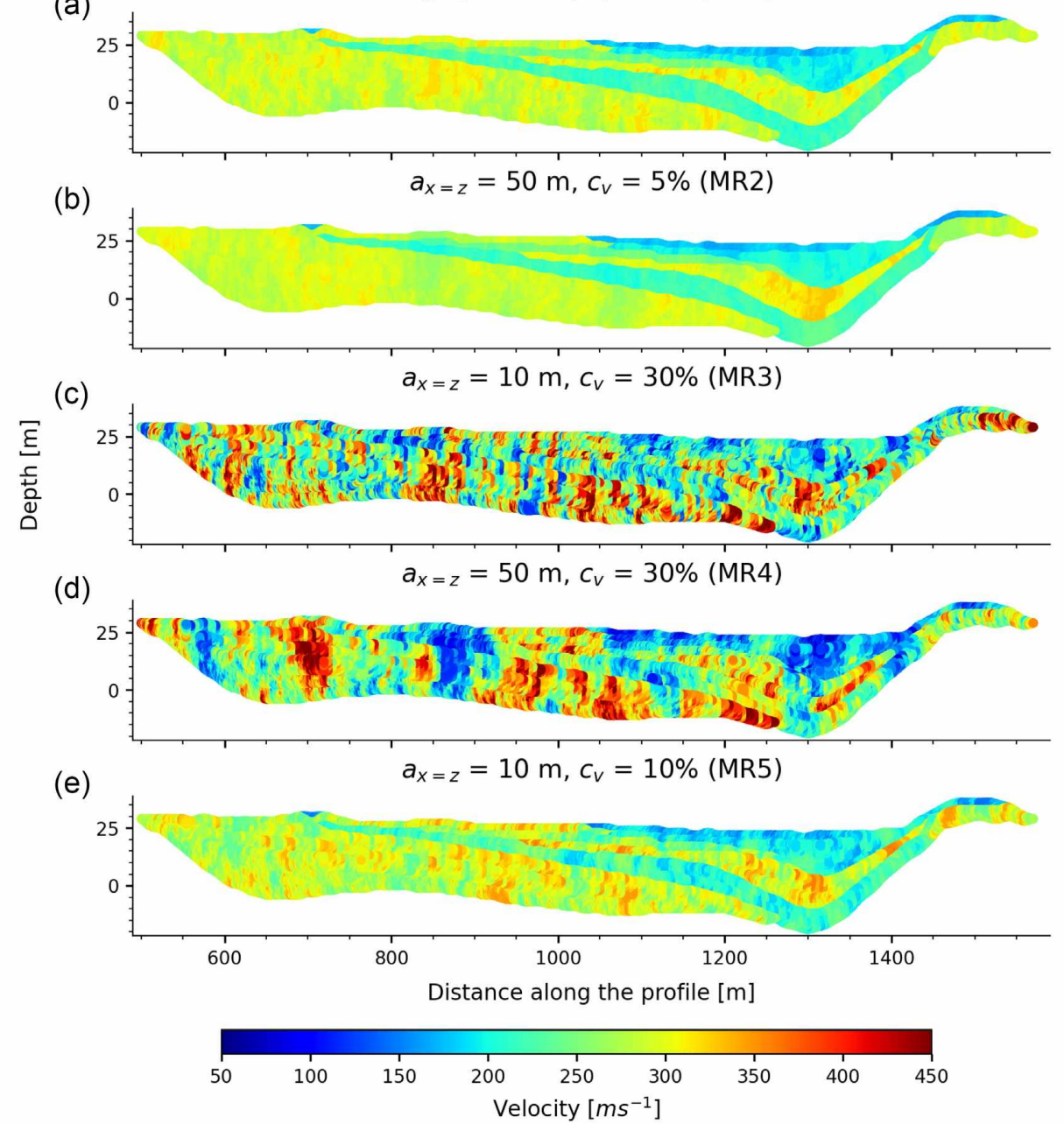

Figure 4. One realization of the random media in Table 2 after interpolation with the basin's spectral element grid for: (a) model R1, (b) model R2, (c) mode1 R3, (d) model R4 and (e) model R5. See Table 2 for the characteristics of each model.

differences. We do not categorize the misfit values into goodnessof-fit (GOF) categories as in Kristekova et al. (2009), but rather interpret them as relative differences between the wavefield computed in the random media and the one computed in the reference model. As a result, the term misfit in the text denotes the difference with respect to the deterministic model.

Fig. 6 shows the EM and PM (top and bottom plots, respectively) averaged over 10 realizations of the random models. The differences are expressed in percentage and the plots are limited on the horizontal axis between 500 and $1500 \mathrm{~m}$ to show only the variations within the basin. The curves display two trends; (1) An increase in the EM and PM values as the $c_{v}$ increases. As a result, the phase and amplitude differences with the reference model for MR3 and MR4 (magenta and green curves) are higher compared to MR5 (cyan curve), which in turn is higher than MR1 and MR2 (red and blue curves, respectively). (2) For a fixed $c_{v}$, both EM and PM values are positively correlated with the random models' correlation length. Implying that increasing the correlation length will, on average, increase the phase and amplitude differences induced by the random velocities.

The average misfits between the 10 realizations of the random models increase between 1100 and $1400 \mathrm{~m}$, where the basin is deepest, hence there is more interaction between the incident wavefield and the random velocities.

\subsection{Spatial distribution of PGV}

Fig. 7 shows the PGV at the surface across the basin for the reference model (black curve) and one realization of random models MR1 to MR5. As before, the horizontal axis is limited between $500 \mathrm{~m}$ and $1500 \mathrm{~m}$ to show the values for the receivers located within the basin only. For a 5 per cent $c_{v}$ (MR1 and MR2, red and blue curves), the PGV values remain close to those of the deterministic case (black curve). As the $c_{v}$ increases, the spatial variability of the PGV values increases. The PGV values are either locally increased or decreased compared to the reference case. A higher spatial variability is observed for models MR3 and MR4 (30 per cent $c_{v}$ ) compared to MR5 (10 per cent $c_{v}$ ). No particular trend is observed when the correlation length varies for fixed $c_{v}$.

We also compute the ratio between each random model and the reference to estimate the factors by which velocity heterogeneities increase or decrease the PGV values (bottom panel of Fig. 7). The ratios for models MR1 and MR2 are approximately one for most receivers and locally vary between 0.8 and 1.3 for the receivers located between 1200 and $1350 \mathrm{~m}$. For MR5, they vary between 0.6 and 1.7. Whereas for MR3 and MR4, they vary between 0.5 and 2.4

To quantify in a different way how the heterogeneities affect the wave propagation, we plot the PGV arrival time $\left(T_{\mathrm{pgv}}\right)$ at each 
(a)

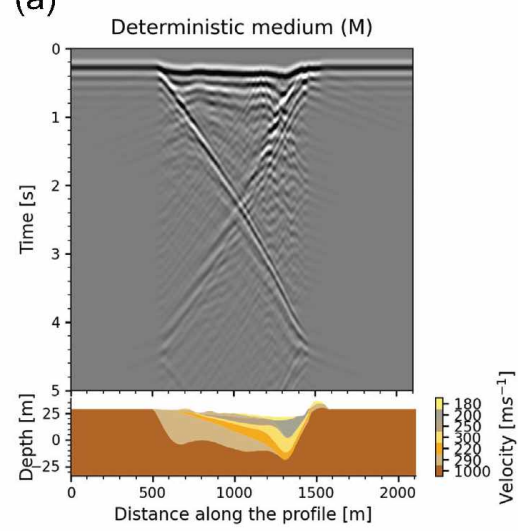

(d)

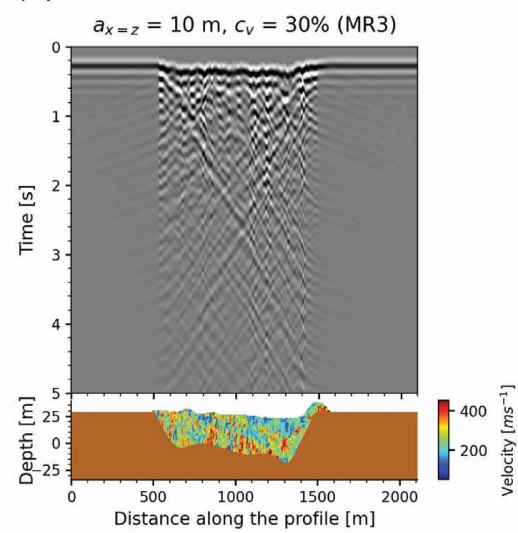

(b)

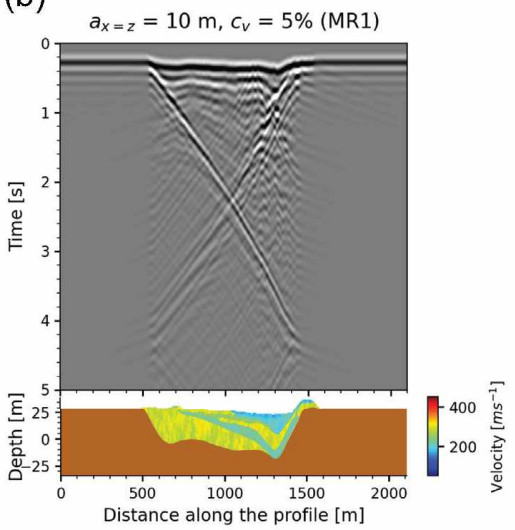

(e)

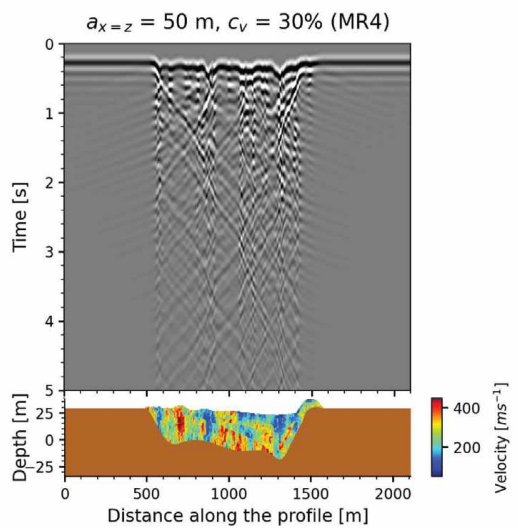

(c)

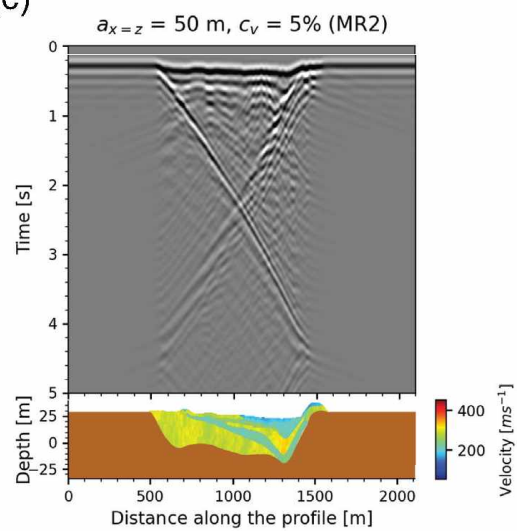

(f)

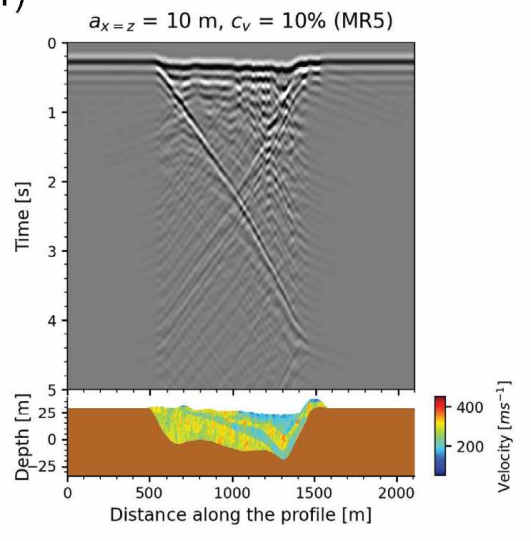

Figure 5. Velocity seismograms (top plots) recorded by the array of surface receivers in (a) the reference model and (b-f) random models MR1 to MR5 (bottom plots). See Table 2 for the characteristics of the random media.
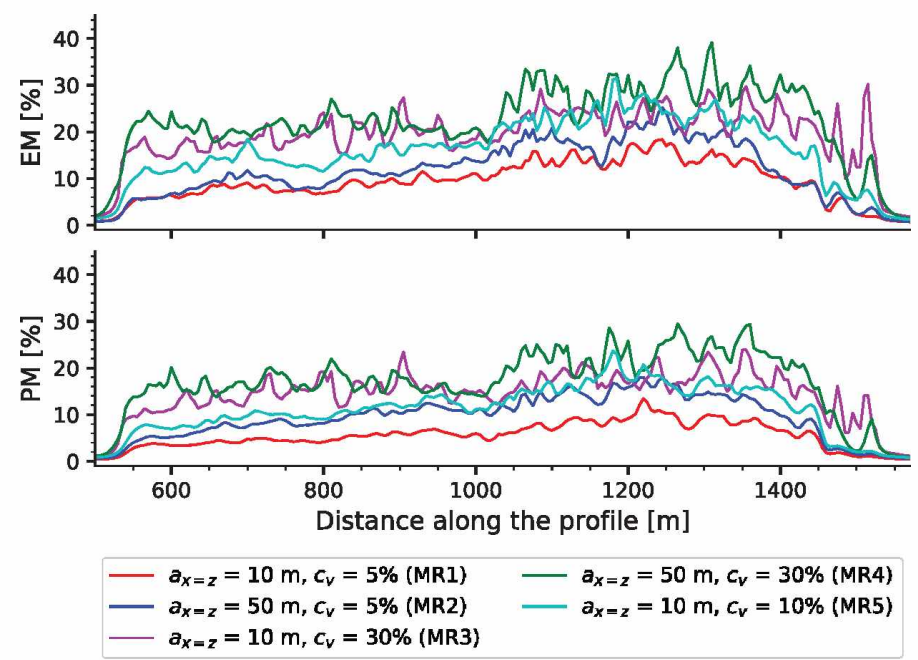

Figure 6. Average envelope misfit (top) and phase misfit (bottom) between the signals of 10 realizations of each random medium (coloured curves) with reference medium.

receiver for both the reference and one realization of the random media in Fig. 8(a). In the deterministic medium (black curve), the highest $T_{\mathrm{pgv}}$ are observed at the basin edges. This is due to basin edge generated waves, which have higher amplitudes and arrive later. The $T_{\text {pgv }}$ is constant at the central receivers because the PGVs are all carried by the direct wave. In models MR1, MR2 and MR5, the pattern remains the same, with local $T_{\mathrm{pgv}}$ at certain receivers between
1250 and 1400 being mildly affected by the velocity heterogeneities. For MR3 and MR4, notable variations in the $T_{\mathrm{pgv}}$ are observed. This is because the velocity fluctuations in these models lead to phase conversions, which can have higher amplitudes than the direct arrivals. The resulting $T_{p g v}$ at these receivers locally differ from the deterministic case because they are carried by different wave trains. This can be seen in Fig. 8(b), where we plot the seismogram for 

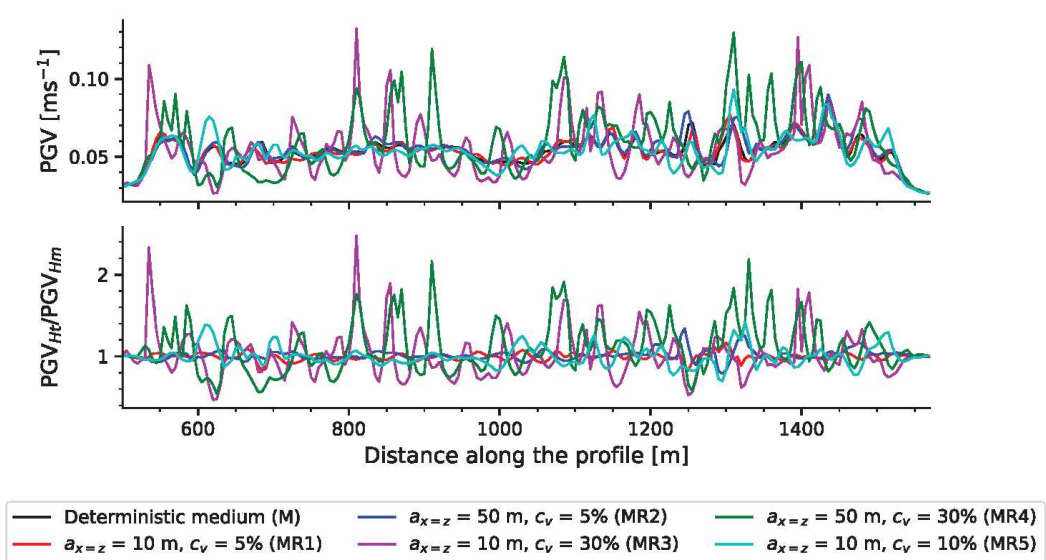

Figure 7. Top: PGV at the surface receivers for the reference medium (black curve) and for a single realization of the random media MR1, MR2, MR3, MR4 and MR5 (red, blue, magenta, green and cyan curves respectively); bottom: ratio between each random models' PGV curves and the reference PGV curve.

(a)

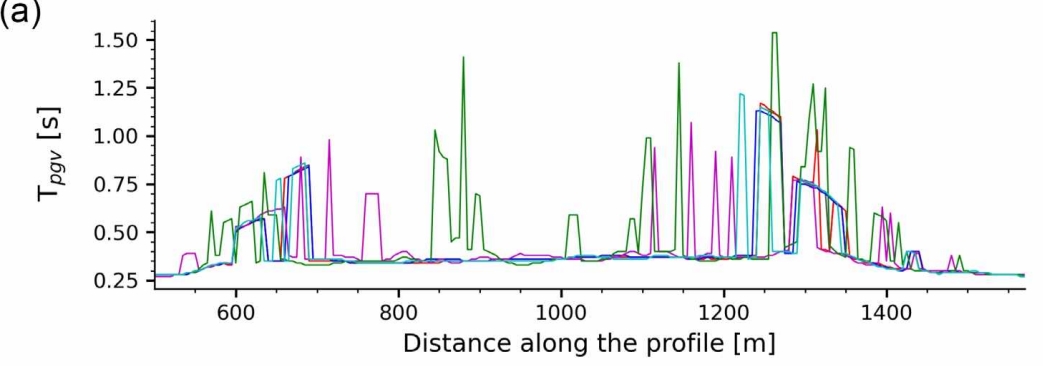

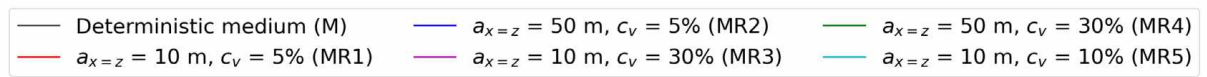

(b)

Receiver at $x=1215 \mathrm{~m}$

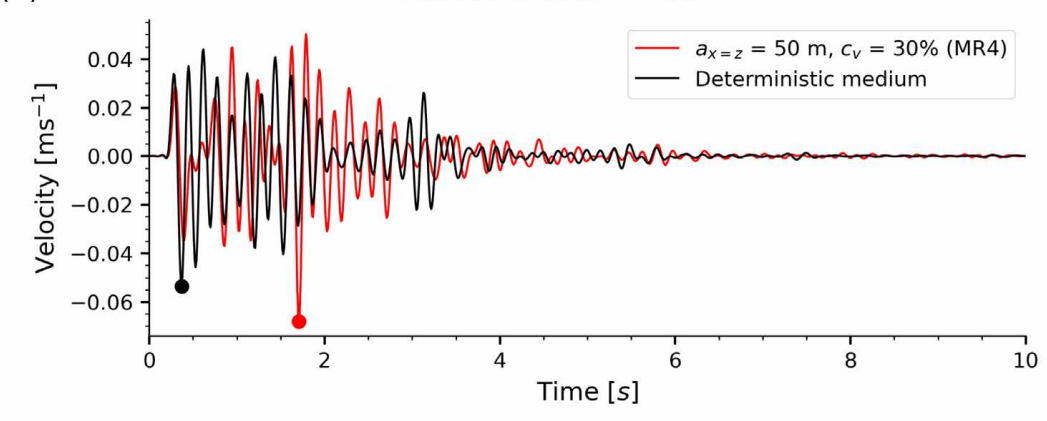

Figure 8. (a) Arrival time of the observed PGV; (b) seismograms of a surface receiver located at $1215 \mathrm{~m}$ on the horizontal profile for the deterministic medium (black signal) and a single realization of medium MR4 (red signal). The black and red dots highlight the PGV on both signals.

a receiver located at $x=1215 \mathrm{~m}$ for the reference medium (black curve) and one realization of model MR4 (red curve). The PGVs (denoted by the black and red dots) in both cases are carried by different wave phases, the first arrival phase for the deterministic trace, and by a later arrival for model MR4.

To have a better picture on how the PGV values vary between the different realizations of the random media, we computed a proxy of the coefficient of variation by taking the ratio between the inner- 68 th percentile and twice the median of the 10 random models (Fig. 9). The curves show that the variability increases with the amount of perturbation of the velocity heterogeneities. On average, the PGV variability varies around 5 per cent for MR1 and MR2, 10 percent for MR5 and 30 per cent for MR3 and MR4, which coincide with the coefficient of variation of the random perturbation. Hartzell et al. (2010) reported similar values of the resulting variability of the PGV to those used as the fluctuations of the velocity model. In our case, this might be a coincidence, and further analyses should be done.

\subsection{Spectral ratios}

The standard spectral ratio or transfer function (TF) is commonly used to assess site effects as it quantifies the spectral amplification of the site (Borchedt 1970). We calculate the 2-D TF of the sedimentary basin by computing the ratio between the Fourier spectrum at each receiver and the average Fourier spectrum of the receivers located on the bedrock. The signals were detrended and bandpass filtered between 0.1 and $10 \mathrm{~Hz}$ before computing their Fourier transform. The Fourier spectra were then smoothed using a Konno-Ohmachi 

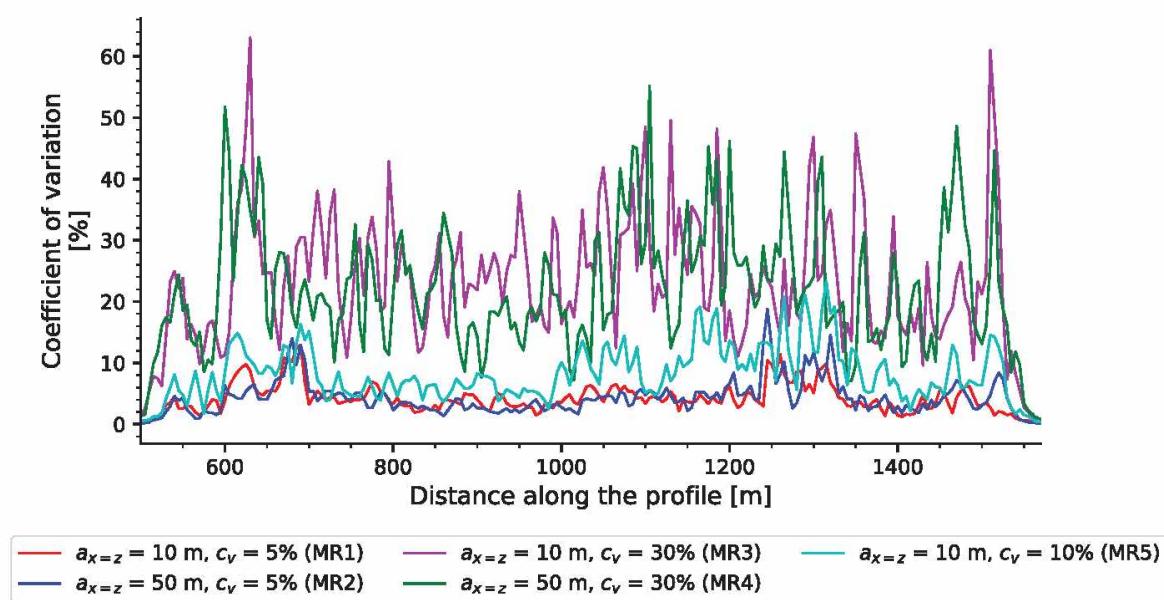

Figure 9. Coefficient of variation of the PGV curves between the 10 realizations of each random model. The variance between the realizations of the random models increases as the $c_{v}$ of the random models.

smoothing function (Konno \& Ohmachi 1998), with a bandwidth of 40. To make the 2-D TFs of the different models comparable, we used the average Fourier spectra of the receivers located on the deterministic model's bedrock as the common denominator for the spectral ratios.

Fig. 10(a) shows the 2-D transfer function of the reference medium. The ordinate axis stands for frequency, and the abscissa axis is the distance along the profile. The colour bar indicates the spectral amplification and is plotted between 1 and 7. The 2-D TF describes the sedimentary basin's seismic response. The amplification at low frequencies $(1-3 \mathrm{~Hz})$ is mainly due to the impedance contrast between the basin and the bedrock. At higher frequencies $(>3 \mathrm{~Hz})$, the amplification is due to a combination of the layer's reverberation, lateral propagation in the basin, and the basin geometry. The basin layers mostly affect the high- frequency amplification between 1100 and $1500 \mathrm{~m}$, where their geometry is more complex. Between 500 and $1100 \mathrm{~m}$, the high-frequency amplifications are the harmonics of the fundamental frequencies.

Figs 10(b) (f) show the 2-D TFs for a single realization of random models MR1 to MR5, respectively. The TFs for MR1 and MR2 show slight differences compared to the reference model, confirming that a small $c_{v}$ of 5 percent has little effect on the spectral amplification of the ground motion. In this case, the properties of the deterministic model dominate the properties of the simulated wavefield, hence the spectral amplification follow similar patterns as the reference case. For MR5, with a $c_{v}$ of 10 per cent, higher fluctuations in the spectral amplification (especially at frequencies higher than $6 \mathrm{~Hz}$ ) are observed compared to MR1 and MR2. However, the overall amplification pattern remains similar to the reference case because the deterministic structure still dominates the velocity model. In contrast, the TFs for MR3 and MR4 display a different seismic response. Moreover, the basin's fundamental frequency is strongly affected by the intense perturbation of the velocity media. The impedance contrast between the sediments and the bedrock is less important than the strong local velocities contrast within the sediments. Furthermore, localized but strong heterogeneities also affect higher resonance frequencies producing an overall broadband spectral amplification. As before, for a constant coefficient of variation, the correlation length has a mild effects on the basin amplification.

\subsection{Pseudo-spectral acceleration}

We compute the response spectra at two particular periods, 0.5 and $0.17 \mathrm{~s}$. The former is close to the resonance frequency $(2 \mathrm{~Hz})$ of the whole basin, while the latter is the predominant frequency $(6 \mathrm{~Hz})$ of the input motion.

We plot in Figs 11(a) and (b) (top panels), for both periods, the proxy of the coefficient of variation between the 10 realizations of each random model and the ratio between the median spectral acceleration and the deterministic model (bottom panels). Models MR1, MR2 and MR5 show no major variability in the computed PSA due to velocity heterogeneities. In contrast, the effect of the velocity heterogeneities is remarkable for MR3 and MR4. Besides, for the same coefficient of variation, the effect of correlation length is little on the PSA's spatial distribution. In general, the coefficient of variation for models MR3 and MR4 at $0.17 \mathrm{~s}$ is larger than the case of $0.5 \mathrm{~s}$. This is expected because the transfer functions also show strong amplifications at those periods in the basin (see Figs $10 \mathrm{~d}$ and e).

The ratio between the median response spectra of 10 random realizations and the reference case (Figs 11a and b, lower panels) present values close to one for small velocity fluctuations (MR1 and MR2, red and blue curves, respectively), while they display high spatial variability for larger perturbations (MR3 and MR4), either amplifying or deamplifying the spectral accelerations, by factors between 0.5 and 1.5 . The spatial variability and amplification factors are higher for the shorter period of the oscillator, which coincides with the input motion's dominant frequency.

\section{DISCUSSION}

This parametric analysis shows that the ground motion indicators and site response are highly sensitive to the random media's coefficient of variation, and to a lesser extent, the correlation length - for this particular geometry configuration. This corroborates the results of El Haber et al. (2019) for wave propagation in a model composed of one heterogeneous layer overlaying a bedrock. An increase of the random medium's coefficient of variation leads to an increase in the ground motion's spatial variability and in the variability of the ground motion intensity measures. 
(a)

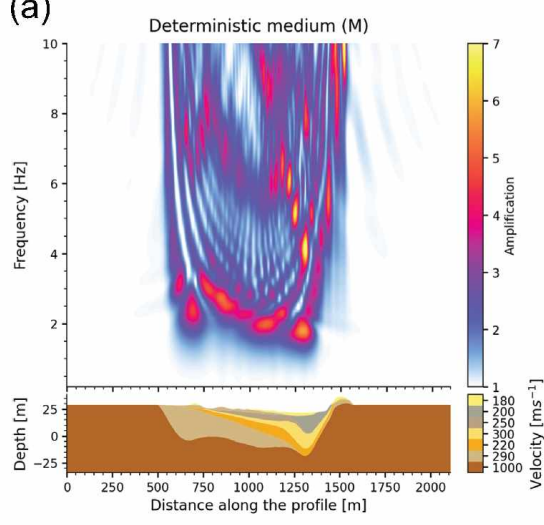

(d)

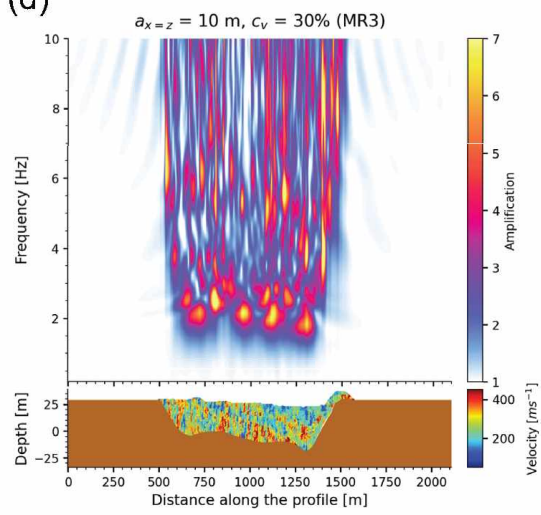

(b)

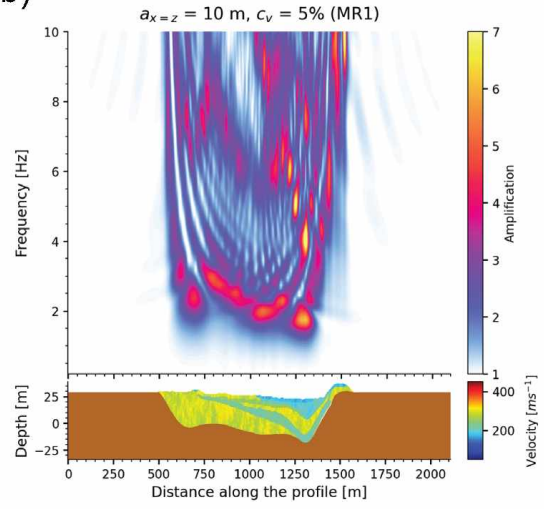

(e)

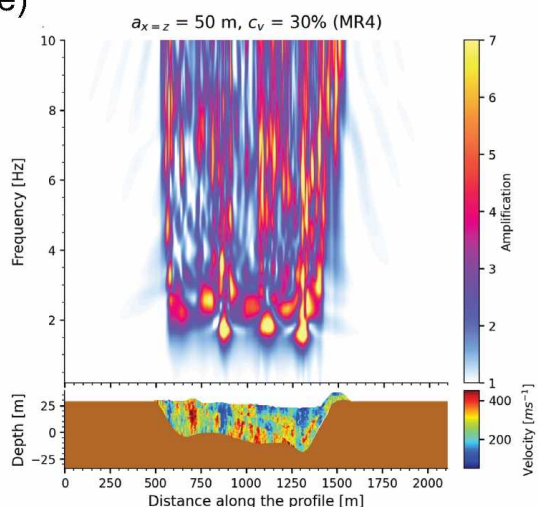

(c)

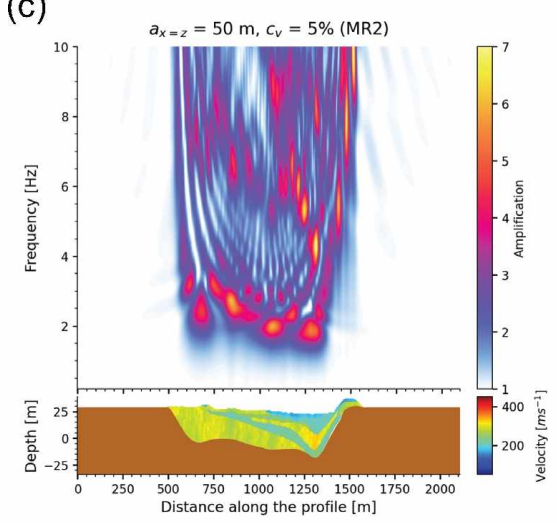

(f)

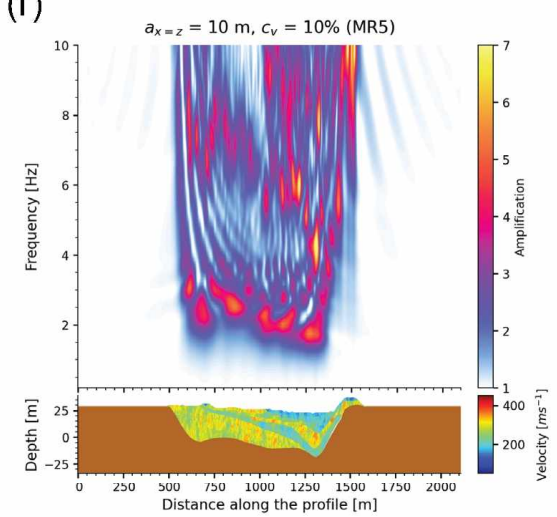

Figure 10. 2-D TF computed in (a) the deterministic velocity model; and one realization of the random models (b) MR1, (c) MR2, (d) MR3, (e) MR4 and (e) MR5. The small amplifications at some frequency bands in the bedrock are due to the leaking waves from the sediments to the bedrock. The figure displays the degree of change in the 2-D amplification patterns and values compared to the deterministic TF (a).

When modelling small-scale velocity heterogeneities using stochastic methods, the effects on wave propagation through a random medium are generally described in terms of statistical averages of many observations (e.g. Korn 1993; Sato \& Fehler 2012). In the context of site effect assessment, the interest is to understand whether averaging several realizations of random media provides physically meaningful results, given that there is only one random realization of the subsurface. Moreover, performing a multitude of simulations for different realizations of random media requires high computational demands. In a practical framework, this raises a practical question on the number of simulations necessary to perform reliable analysis.

In our study, we consider 10 random media models. We plot in Figs 12 and 13 the geometric mean of the 2-D TFs and the associated coefficient of variation between the 10 stochastic realizations. We compute the geometric mean instead of the arithmetic mean because it is conventionally on observed data to obtain the average of spectral ratios, which can strongly vary from one earthquake to another. However, in this study the geometric and arithmetic mean have no major differences in their values.

For models MR1 and MR2 (Figs 13a and b), the variance between the TFs of the 10 realizations is low $(<20$ per cent $)$ and their averages are comparable to the reference case (Fig. 10a). For model MR5 (Fig. 13e), there is a slight increase in the variance for frequencies higher than $5 \mathrm{~Hz}$. However, the average TF for MR5 (Fig. 12e) remains close to the reference case. For these models the deterministic structure controls the wave propagation and, therefore, their average TFs follow similar patterns as the reference case.
On the contrary, the large coefficient of variation between the TFs of models MR3 and MR4 (Figs 13c and d) leads to a smoother average response compared to the reference case and individual realizations. The decrease in the mean amplification is because the spatial distribution of the velocity heterogeneities strongly varies from one realization to another. Therefore, averaging the TFs of the 10 realizations produces a smoothing effect on the amplification, leading to an average TF that largely differs from a single realization. We note that models MR3 and MR4 correspond to extreme cases of velocity perturbations in which the deterministic structure is completely destroyed. Although values of 30 per cent coefficient of variation can be observed in sediments (e.g. Wills \& Clahan 2006; Sato 2019), we decide to use models MR3 and MR4 in our analysis to study the effects for extreme cases of velocity perturbations in sediments. The smoothing of the average amplification in these media suggests that the average TF between the stochastic realizations of the random models could be biased due to the presence of extreme results for a given realization. We propose to use percentiles in addition to the mean to describe the site response in order to capture the associated uncertainties. Figs 14(a) and (b) show the 16 th and 84 th percentile, respectively, for model MR3. We can clearly see that the variability in the amplification is important. A better visualization of this variability can be obtained by plotting the TF at a single surface receiver. Fig. 14(c) shows the site amplification at a surface receiver located at $1215 \mathrm{~m}$ on the horizontal profile. The 84th percentile shows, for example, a resonance peak between 3 and $4 \mathrm{~Hz}$ that is not clearly visible on the median and mean curves. 
(a)
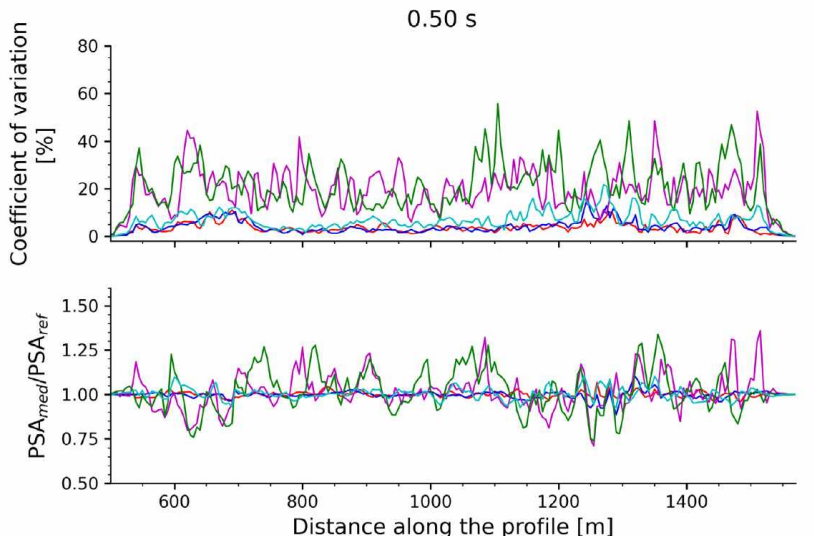

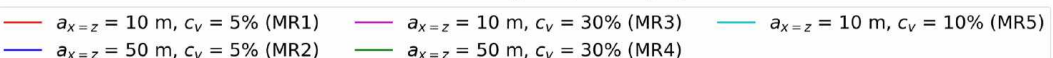

(b)
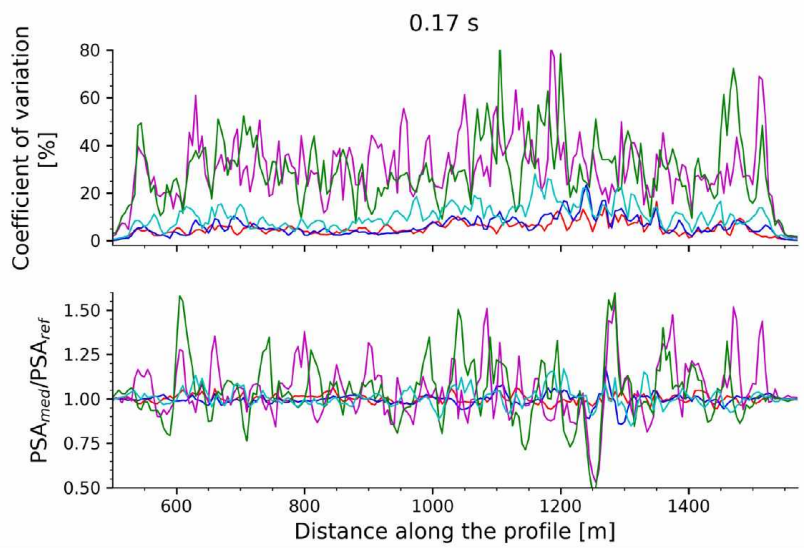

Distance along the profile $[\mathrm{m}]$

$-a_{x=z}=10 \mathrm{~m}, c_{v}=5 \%$ (MR1) - $a_{x=z}=10 \mathrm{~m}, c_{v}=30 \%$ (MR3) - $a_{x=z}=10 \mathrm{~m}, c_{v}=10 \%$ (MR5)

$-a_{x=z}=50 \mathrm{~m}, c_{v}=5 \%($ MR2 $)-a_{x=z}=50 \mathrm{~m}, c_{v}=30 \%($ MR4)

Figure 11. Top: coefficient of variation of the PSA between the 10 realizations of each random model at the periods (a) $0.5 \mathrm{~s}$ and (b) $0.17 \mathrm{~s}$. Bottom: the ratio between median PSA of the random models and the detertiministic model's PSA.

The choice of the parameters of the ACF in this analysis is mainly based on values reported in the literature for sediments (e.g. Wills \& Clahan 2006; Thompson et al. 2009; Sato 2019). Model MR5, with a $c_{v}$ of 10 per cent, was chosen as an intermediate case which still preserves the deterministic feature of velocity model. Models MR3 and MR4 with $c_{v}$ values of 30 per cent were extreme cases that destroy the deterministic structure of the basin and lead to strong effects on the intensity measures. The synthesis of the estimates of $c_{v}$ in sediments in table 4 of Thompson et al. (2009) shows that a $c_{v}$ of 30 per cent is more probable to explain the observations in sediments. Whether velocity fluctuations should preserve or not the deterministic feature of a basin is an open question. The coefficient of variation in a given layer depends on its geological history and properties of its material. We cannot justify that the choice of a $c_{v}$ value should be limited by any velocity contrast with other layers. This point stresses the importance of the resolution of the spatial characterization of the velocity heterogeneities from in-situ data.

This parametric analysis also shows that the effects of the random velocities are different on the frequency and time domain intensity measures. In the frequency domain, the transfer function, is independent of the source time function but depends on the impedance contrast present in the velocity model. Hence, as long as the velocity heterogeneities do not change the deterministic structure of the velocity model (e.g, models MR1, MR2 and MR5), the overall spectral amplification (Figs 10b, $\mathrm{c}$ and $\mathrm{f}$ ) will remain similar to the reference model. Whereas, when they strongly modify the impedance contrasts such as in models MR3 and MR4, the spectral amplification becomes completely different from the reference case (Figs $10 \mathrm{~d}$ and e). In the time-domain, the PGV is affected through constructive or destructive interferences between waves generated by local velocity heterogeneities and other waves that propagate inside the basin. As a result, a high spatial variability in their values is observed as the intensity of the velocity heterogeneities increases (Figs 9 and 11). More generally, these interferences depend on the source time function and cannot be seen in the frequency domain. Therefore, both frequency and time domain parameters are useful to understand the effect of velocity fluctuations on the ground motion.

In this study, we assumed that layers have the same random media characteristics to avoid abrupt changes in velocity at the layer's interface. Such an assumption is questionable and we could also have generated one random medium per layer while keeping the same Von Karman ACF properties in each layer. This could have led to stronger velocity contrast between layers since each layer is considered independently. Some preliminary tests have shown that we obtained similar results in both cases. However, this should be further studied but this is beyond the scope of this study. 
(a)

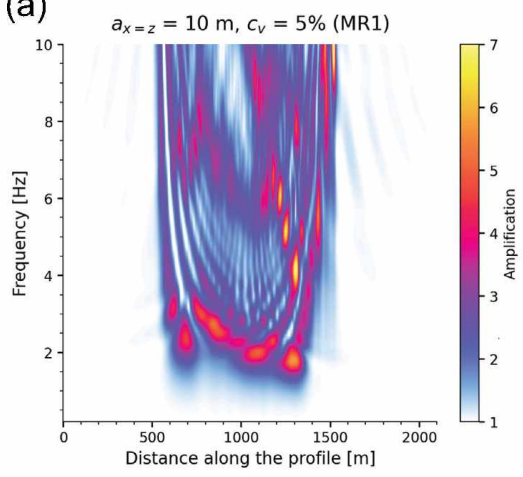

(d)

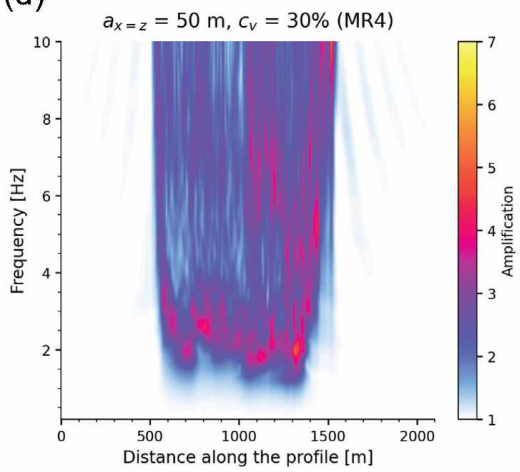

(b)

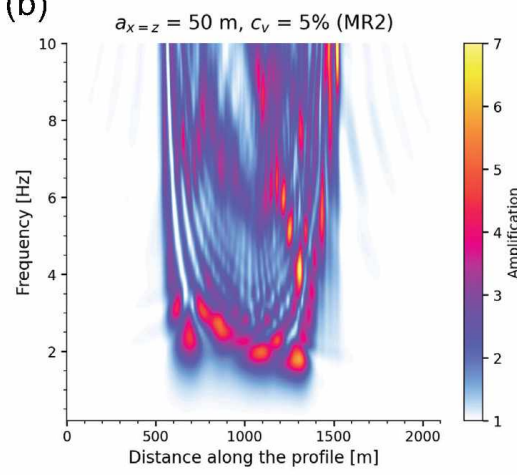

(e)

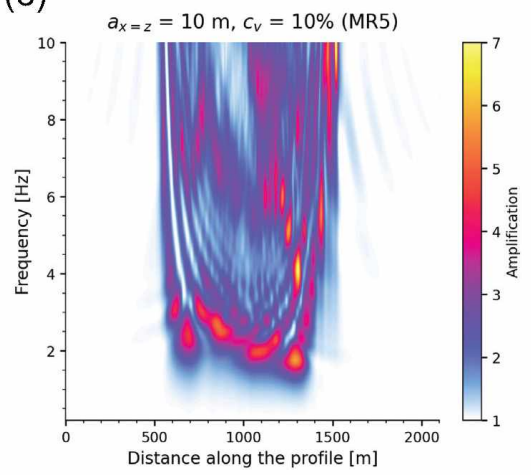

(c)

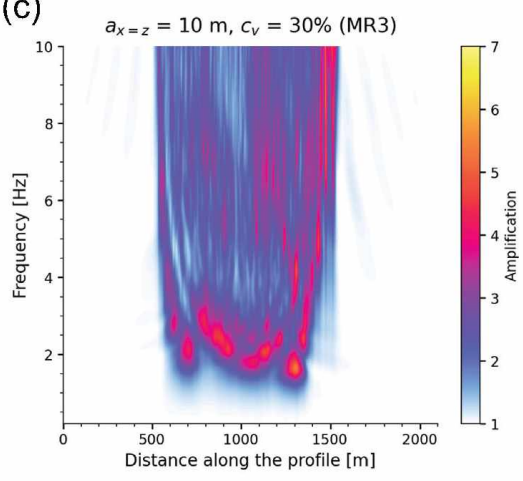

Figure 12. TF Geometric mean between the 10 realizations of the random models (a) MR1, (b) MR2, (c) MR3, (d) MR4 and (e) MR5. Note the smoothing effect on the amplifications due to averaging, which leads lower amplifications (especially for media MR3 and MR4) compared to the reference case (Fig. 10a) and models with lower coefficient of variation (MR1, MR2 and MR5).

(a)

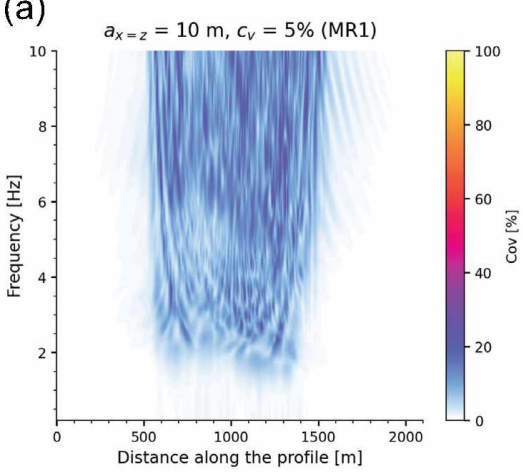

(d)

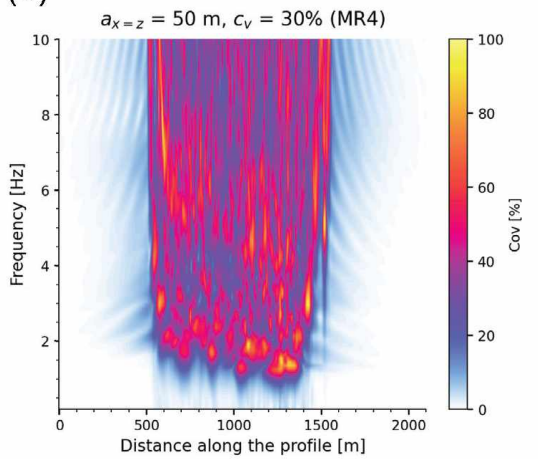

(b)

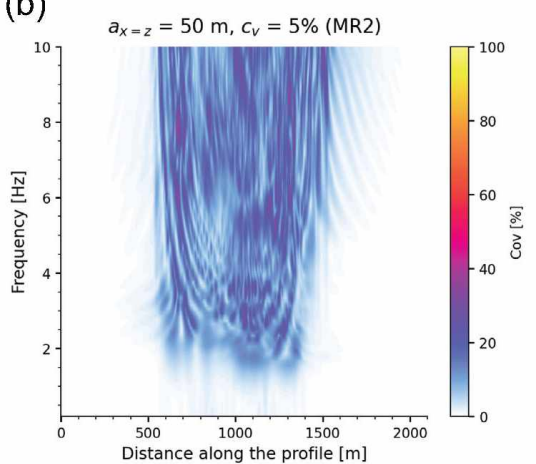

(e)

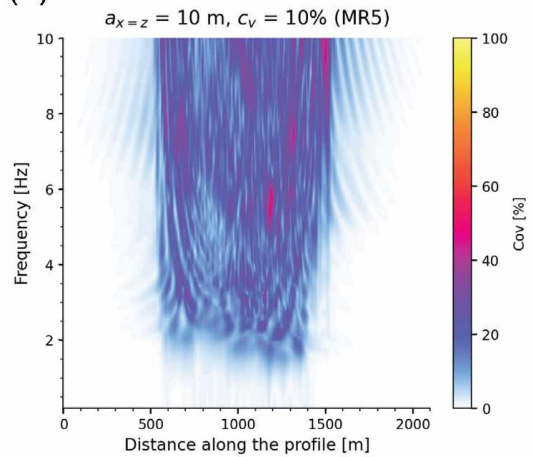

(c)

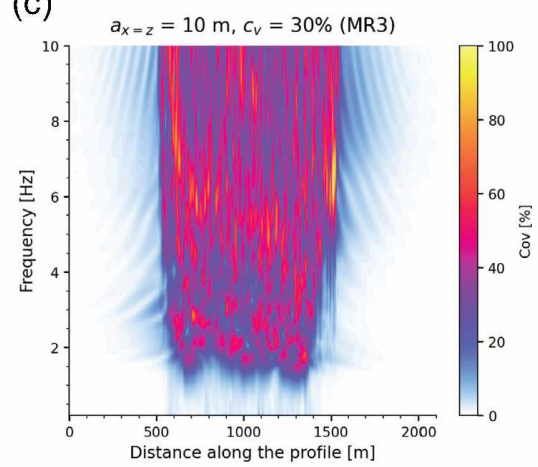

Figure 13. Coefficient of variation (Cov) between the TFs of the 10 realizations of the random models (a) MR1, (b) MR2, (c) MR3, (d) MR4 and (e) MR4. A higher variance in the TFs is observed for media MR3 and MR4 with a $c_{\gamma}$ of 30 per cent. 
(a)

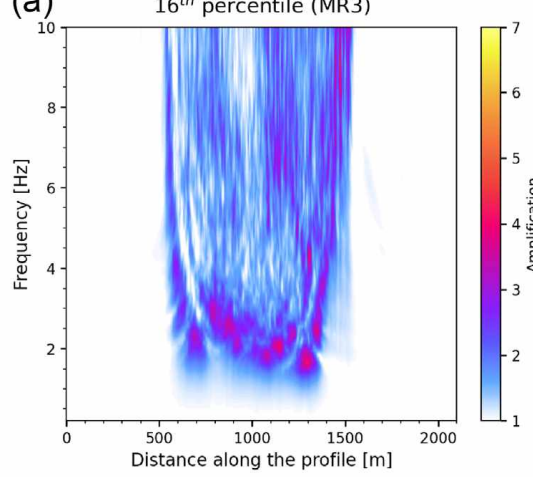

(b)

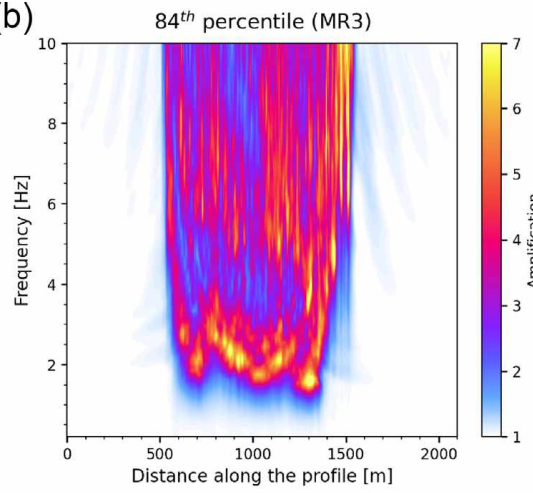

(c)

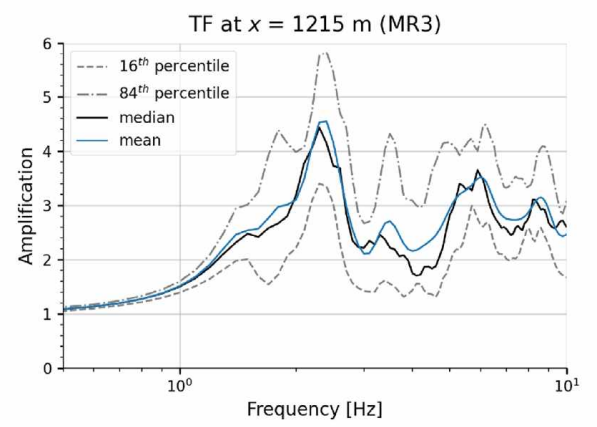

Figure 14. The 16th percentile (a) and 84th percentile (b) between the TFs of the 10 realizations of model MR3. (c) Plot showing 16 th percentile, 84 th percentile, median and mean at the point $\mathrm{x}=1215 \mathrm{~m}$ on the horizontal profile.

\section{CONCLUSION S}

We perform a series of numerical simulations to investigate how velocity heterogeneities affect seismic ground motion in a complex sedimentary basin. The velocity heterogeneities are modelled as a stationary random process following a Von Karman ACF. We vary the coefficient of variation and the correlation length of the ACF to analyse parametrically how they affect the ground motion and related intensity measures.

The presence of velocity heterogeneities in the basin mainly scatter the late arrivals and to a fewer extent the direct waves; hence affecting the resulting ground motion intensity measures. The simulations show that the envelope and phase misfits (EM and PM), between the wavefields of the random and deterministic media, become large as the coefficient of variation of the random velocities increases. This increase is spatially correlated with the presence of low-velocity zones in the random models. The differences also seem to augment with an increase of the random media's correlation length, although less pronounced. This is probably because the size of the basin is relatively small; thus, there is not enough propagation time for their effect to be seen on the travel times.

Regarding the PGV, the presence of random velocities leads to an extended spatial variability, which increases with the coefficient of variation of the velocity fluctuations. The results also show that the PGV values are very sensitive to the random media's realization, as evidenced by the high variability in the PGV computed between the 10 realizations. This is also reported by Hartzell et al. (2010) for a 3 -D wave propagation in random media in the area of San Francisco. Similar trends are observed for the pseudo-spectral acceleration.

The effect of the random velocity heterogeneities on the 2-D spectral ratios is less pronounced for the random media with 5 per cent coefficient of variation. Whereas for the 30 per cent case, the basin seismic response becomes quite different from the reference model. We also observe a reduction of the maximum amplitude of the mean spectral ratios that is more pronounced with a coefficient of variation of 30 per cent. This reduction is also reported in the studies by Assimaki et al. (2003) and El Haber et al. (2019), and it is due to a smoothing effect in the averaging of the spectral ratios of different realizations of random media. The large variance in the site response associated to the media with high velocity fluctuations suggests that a higher number of realizations of the random media are required to describe a central tendency. For a limited number of simulations, we recommend using the percentiles of the distribution in addition to the mean and standard deviation to describe the site response in random media.
A peculiar characteristic of the Nice sedimentary basin is its complex geometry and the absence of strong impedance contrast between the sediments due to their close velocities. As a result, large coefficients of variation of the random models can easily destroy the initial deterministic structure. Exploring the effect of random velocities on layers having very different velocities should be a natural continuation of this study.

These results show that the effect of random velocity fluctuations on ground motion indicators is important, as they increase their variability. The main controlling factor, in this particular case, is the coefficient of variation of the velocity fluctuation. The differences in the values of ground motion intensity measures for the different configurations of the random media highlight the importance of a fine characterize of soil properties in a site.

This study is by no means exhaustive, and the present conclusions may not be generalized to all cases. Our results show high complexities in the computed ground motion despite the simplifications in the modelling assumptions - we considered isotropic random media and assumed that all the sedimentary layers have the same velocity perturbation, which may not be true in reality. The simulations were performed using a wavelet with a given dominant frequency as a source time function. As next steps, it would be necessary to perform the analysis using a realistic source time functions, having a broadband frequency content, to conjointly investigate the effects of the source time function and medium properties. It would also be interesting to consider the case of $P-S V$ polarized waves and to model the velocity heterogeneities using anisotropic random media.

\section{ACKNOWLEDGEMENTS}

The authors would like to thank the two anonymous reviewers for their thoughtful and insightful comments that helped improve the quality of this manuscript. The authors also thank Etienne Bertrand for providing us with the 2-D velocity model of the sedimentary basin. We also thank Emmanuel Chaljub and Jean-Paul Ampuero for constructive discussions and Walter Imperatori for constructive discussions about the generation of random media. We thank Elif Oral for providing us with her version of the numerical code SEM2DPACK used in this study. The simulations were performed on the IRSN ACALU computer cluster.

\section{DATA AVAILABILITY}

The simulations data underlying this paper will be shared upon reasonable request to the corresponding author. 


\section{REFERENCES}

Ampuero, J., 2003. Etude physique et numérique de la nucléation des séismes, $P h D$ thesis, Université Paris 7, Denis Diderot, Paris.

Anderson, J., Bodin, P., Brune, J., Prince, S., Quaas, R. \& Onate, M., 1986. Strong ground motion from the the Michoacan, Mexico, earthquake, Science, 233, 1043-1049.

Arnal, C. \& Martin, R., 1998. Projet gemitis-nice: evaluation des dommages directs d'un séisme sur la ville nice, scénario de risque, extension des résultats au départements des alpes maritimes., Tech. rep., BRGM, Rapport BRGM n ${ }^{\circ} 40612$.

Assimaki, A., Pecker, A., Popescu, R. \& Prevost, J., 2003. Effects of spatial variability of soil properties on surface ground motion, J. Earthq. Eng, 7, 1-44.

Bard, P. et al., 2005. GEMGEP, le risque sismique á Nice. Apport méthodologique, résultats et perspectives opérationnelles, Tech. rep., CETE Méditerranée - LCPC, Rapport final pour la communauté Nice Côte d'Azur.

Bard, P.-Y., 1999. Microtremor measurements: a tool for site effect estimation? Eff. Surf. Geol. Seism. motion, 3, 1251-1279.

Bard, P.-Y. \& Bouchon, M., 1985. The two-dimensional resonance of sediment-filled valleys, Bull. seism. Soc. Am., 75, 519-541.

Bertrand, E., Duval, A.-M., Castan, M. \& Vidal, S., 2007. 3D geotechnical soil model of Nice, France, inferred from seismic noise measurements for seismic hazard assessment, in Proceedings of the AGU Fall Meeting, San Francisco.

Borchedt, R., 1970. Effects of local geology on ground motion near San Francisco Bay, Bull. seism. Soc. Am., 60, 29-61.

Carpentier, S. \& Roy-Chowdhury, K., 2007. Underestimation of scale lengths in stochastic fields and their seismic response: a quantification exercise, Geophys. J. Int., 169, 547-562.

Chaljub, E., Moczo, P., Tsuno, S., Bard, P.-Y., Kristek, J., Kaser, M., Stupazzini, M. \& Kristekova, M., 2010. Quantitative comparison of four numerical predictions of 3D ground motion in the Grenoble valley, Bull. seism. Soc. Am., 100(4), 1427-1455.

Courboulex, F. et al., 2007. Seismic hazard on the French Riviera: observations, interpretations and simulations, Geophys. J. Int., 170, 387-400.

Day, S.M., Bielak, J., Dreger, D., Graves, R., Larsen, S., Olsen, K.B. \& Pitarka, A., 2005. 3D ground motion simulations in basins: final report for lifelines project 1a03, Tech. Rep., Pacific Earthquake Engineering Research Center.

El Haber, E., Cornou, C., Jongmans, D., Youssef Abdelmassih, D. \& LopezCaballero, F., 2019. Influence of 2D heterogeneous elastic soil properties on surface ground motion spatial variability, Soil Dyn. Earthq. Eng., 123, $75-90$.

Emmerich, H. \& Korn, M., 1987. Incorporation of attenuation into timedomain computations of seismic wave fields, Geophysics, 52(9), 12521264.

Emoto, K., Saito, T. \& Shiomi, K., 2017. Statistical parameters of random heterogeneity estimated by analysing coda waves based on finite difference method, Geophys. J. Int., 211, 1575-1584.

Frankel, A., 1989. A review of numerical experiments on seismic wave scattering, Pure appl. Geophys., 131(4), 639-685.

Frenje, L. \& Juhlin, C., 2000. Scattering attenuation: 2D and 3D finite difference simulations vs. theory, $J$. Appl. Geophys., 41(1), 33-46.

Goff, J. \& Jordan, T., 1988. Stochastic modeling of seafloor morphology: inversion of sea beam data for second-order statistics, $J$. geophys. Res., 93, $13589-13608$.

Hartzell, S., Harmsen, S. \& Frankel, A., 2010. Effects of 3D random correlated velocity pertubations on predicted ground motions., Bull. seism Soc. Am., 100, 1415-1426.

Holliger, K. \& Levander, A., 1992. A stochastic view of lower crustal fabric based on evidence from the Ivrea Zone, Geophys. Res. Lett, 19(11), 11531156.

Holzer, T.L., Bennett, M.J., Noce, T.E. \& Tinsley, J.C., 2005. Shear wave velocity of surficial geologic sediments in northern California: statistical distributions and depth dependence, Earthq. Spectra, 21, 161-177.

Hong, T.-K. \& Kennett, B., 2003. Scattering attenuation of 2D elastic waves: theory and numerical modeling using a wavelet-based method, Bull. seism. Soc. Am., 93(2), 922-938.
Imperatori, W. \& Mai, P., 2013. Broad-band near-field ground motion simulations in 3-dimensional scattering media, Geophys. J. Int, 192, 725-744.

Imperatori, W. \& Mai, P., 2015. The role of topography and lateral velocity heterogeneities on near-source scattering and ground-motion variability, Geophys. J. Int., 202, 2163-2181.

Ishimaru, A., 1978. Wave Propagation and Scattering in Random Media, Academic Press, Inc.

Iwaki, A., Maeda, T., Morikawa, N., Takemura, S. \& Fujiwara, H., 2018. Effects of random $3 \mathrm{D}$ upper crustal heterogeneity on longperiod $(\geq 1 \mathrm{~s})$ ground-motion simulations, Earth Planets Space, 70, doi:10.1186/s40623-018-0930-5

Kanai, K., 1993. Engineering Seismology, University of Tokyo Press.

Klimes, L., 2002. Correlation functions of random media, Pure appl. geophys., 159, 1811-1831.

Konno, K. \& Ohmachi, T., 1998. Ground motion characteristics estimated from spectral ratio between horizontal and vertical components of microtremors, Bull. seism. Soc. Am., 81, 228-241.

Korn, M., 1993. Seismic waves in random media, J. Appl. Geophys., 29, $247-269$.

Kristekova, M., Kristek, J., Moczo, P. \& Day, S., 2006. Misfit criteria for quantitative comparison of seismograms, Bull. seism. Soc. Am., 96, 18361850.

Kristekova, M., Kristek, J. \& Moczo, P., 2009. Time-frequency misfit and goodness-of-fit criteria for quantitative comparison of time signals, Geophys. J. Int, 178, 813-825.

Ktenidou, O.-J., Chávez-García, F.-J., Raptakis, D. \& Pitilakis, K., 2016. Directional dependence of site effects observed near a basin edge at Aegion, Greece, Bull. Earthq. Eng., 14(3), 623-645.

Liu, P. \& Archuleta, R., 2006. Efficient modeling of $Q$ for 3D numerical simulation of wave propagation, Bull. seism. Soc. Am., 96, 1352-1358.

Moczo, P., J. K. \& Galis, M., 2014. The Finite-Difference Modeling of Earthquake Motions Waves and Ruptures, Cambridge Univ. Press.

Nakamura, Y., 1989. A method for dynamic characteristics estimation of subsurface using microtremor on the ground surface, $Q$. Rep. Railw. Tech. Res. Inst., 30, 25-33.

Nakata, N. \& Beroza, G., 2015. Stochastic characterization of mesoscale seismic velocity heterogeneity in Long Beach, California, Geophys. J. Int., 203, 2049-2054.

Oral, E., Gélis, C. \& Bonilla, L., 2019. 2-D P-SV and $S H$ spectral element modelling of seismic wave propagation in non-linear media with porepressure effects, Geophys. J. Int., 217(2), 1353-1365.

Pagliaroli, A., Moscatelli, M., Raspa, G. \& Naso, G., 2014. Seismic microzonation of the centeral archaelogical area of Rome: results and uncertainties, Bull. Earthq. Eng., 12, 1405-1428.

Perron, V., Gélis, C., Bérénice, F., Hollender, F., Bard, P.-Y., Cultrera, G. \& Cushing, E., 2018. Can broad-band earthquake site responses be predicted by the ambient noise spectral ratio? Insight from observations at two sedimentary basins, Geophys. J. Int., 215(2), 1442-1454.

Peyrusse, F., Glinsky, N., Gelis, C. \& Lanteri, S., 2013. A nodal discontinuous Galerkin method for site effect assessment in viscoelastic mediaverification and validation in the Nice basin, Geophys. J. Int, 35, 12111223.

Pitarka, A., Trikura, K., Iwata, T. \& Sekiguchi, H., 1998. Three-dimensional simulation of the near-fault ground motion for the 1995 Hyogo-ken Nanbu (Kobe), Japan, earthquake, Bull. seism. Soc. Am., 88(2), 428-440.

Roth, M. \& Korn, M., 1993. Single scattering-theory versus numerical modeling in 2D random-media, Geophys. J. Int., 112(1), 124-140.

Sato, H., 2019. Power spectra of random heterogeneities in the solid earth, Solid Earth, 10, 275-292.

Sato, H. \& Fehler, M., 2012. Seismic Wave Propagation and Scattering in the Heterogeneous Earth, Springer.

Stripajova, S., Moczo, P., Kristed, J., Bard, P.-Y., Hollender, F. \& Sicilia, D., 2018. Extensive numerical study on identification of key structural parameters responsible for site effects, in 16th European Conference on Earthquake Engineering, Thessaloniki.

Tchawe, F.N., Froment, B., Campillo, M. \& Margerin, L., 2019. On the use of the coda of seismic noise autocorrelations to compute H/V spectral ratios, Geophys. J. Int., 220(3), 1956-1964. 
Thompson, E.M., Baise, L.G. \& Kayen, R.E., 2007. Spatial correlation of shear-wave velocity in the San Francisco Bay Area sediments, Soil Dyn. Earthq. Eng., 27, 144-152.

Thompson, E.M., Baise, L.G., Kayen, R.E. \& B, G.B., 2009. Impediments to predicting site response: seismic property estimation and modeling simplification, Bull. seism. Soc. Am., 99(5), 2927-2949.

Trelis, 2019. Trelis (version 16.4) [computer software]. csimsoft, American Fork, UT.
Trifunac, M. \& Lee, V., 1971. Analysis of the Pacoima dam accelerogramSan Fernando, California, earthquake of 1971, Bull. seism. Soc. Am., 61, 1393-1411.

Turker, B., King, J., Hatzfeld, D. \& Nersesov, I., 1984. Observations of hard-rock site effects, Bull. seism. Soc. Am., 74, 121-136.

Wills, C.J. \& Clahan, K.B., 2006. Developing a map of geologically defined site-condition categories for California, Bull. seism. Soc. Am., 96, 14831501. 